\title{
Deriving effective sunspot temperatures from SOHO/VIRGO irradiance measurements ${ }^{\star}$
}

\section{A starspot modelling approach}

\author{
Z. Eker ${ }^{1}$, P. N. Brandt ${ }^{2}$, A. Hanslmeier ${ }^{3}$, W. Otruba ${ }^{4}$, and C. Wehrli ${ }^{5}$ \\ 1 King Saud University, Department of Physics and Astronomy, PO Box 2455, Riyadh, Saudi Arabia \\ 2 Kiepenheuer-Institut für Sonnenphysik, 79104 Freiburg, Germany \\ e-mail: pnb@kis.uni-freiburg.de \\ ${ }^{3}$ Institut für Geophysik, Astrophysik und Meteorologie, $8010 \mathrm{Graz}$, Austria \\ ${ }^{4}$ Sonnenobservatorium Kanzelhöhe, 9521 Treffen, Austria \\ 5 Physikalisch-Meteorologisches Observatorium Davos, WRC, 7260 Davos Dorf, Switzerland
}

Received 1 March 2002 / Accepted 21 March 2003

\begin{abstract}
A method is developed to obtain mean effective sunspot temperatures from satellite irradiance deficits. Using a formalism derived from a star spot modelling approach, this method only requires accurate positions and areas of sunspots - but not intensities. The method is applied to SOHO/VIRGO spectral solar irradiance (SSI) at 402, 500, and $862 \mathrm{~nm}$ as well as total solar irradiance (TSI) measured during the period 16 July to 17 August 1996, the disk passage of active region NOAA 7981. MDI full disk white-light images are used to extract sunspot positions and areas. First, using the spot pixels, which are identified on MDI images as $I_{\mathrm{s}}<0.92$, we find the mean effective temperature of NOAA 7981 to be $\simeq 300 \mathrm{~K}$ less than the local photospheric temperature. Because the area is dominated by penumbral pixels, the temperature is close to a value representing the penumbra. Second, separating umbral and penumbral pixels, we obtain a temperature difference of $\simeq 850 \mathrm{~K}$ for the umbra and of $\simeq 200 \mathrm{~K}$ for the penumbra. Finally, if a $5 \%$ binning of the spot intensities is applied, a temperature deficit of $\simeq 1000 \mathrm{~K}$ is found for the darkest regions of the spot.
\end{abstract}

Key words. Sun: sunspots - Sun: activity - Sun: photosphere - stars: starspots - Sun: UV radiation

\section{Introduction}

The variability of solar irradiance was observationally confirmed during the early eighties by the high precision measurements of the Earth Radiation Budged (ERB) experiment (Kyle et al. 1994) on the NIMBUS-7 satellite which was operated between 1978 and 1993, and by the Active Cavity Radiometer Irradiance Monitoring I (ACRIM I) experiment on the Solar Maximum Mission (SMM) satellite which was operated from 1980 to 1989 (Willson \& Hudson 1991). According to the reviews by Fröhlich (1977), Newkirk (1983), Chapman (1987), Hudson (1988), Fröhlich et al. (1991), and Fröhlich (1994) the short-time changes on time scales of days to months are associated with the evolution of active regions through the combined effect of dark sunspots and bright faculae.

Foukal (1981), Hudson et al. (1982), and Sofia et al. (1982) were pioneers suggesting algorithms to compute theoretical irradiance curves for comparison with actual observations. The

\footnotetext{
Send offprint requests to: Z. Eker, e-mail: eker@ksu.edu.sa

$\star$ Appendixes A, B are only available in electronic form at http://www . edpsciences.org
}

computed function is called photometric sunspot index (PSI). The photometric facular index (PFI) later was included in irradiance models by Chapman et al. (1982), Chapman (1984), and Chapman \& Meyer (1986). Those early models use constant contrasts for the spots and the faculae. Brandt et al. $(1990,1992)$ have shown that both the umbra-penumbra area ratio $(0.32)$ and the bolometric contrast $(0.315)$ used in those models need improvements. Thus, instead of the classical PSI, a new improved APSI (Area dependent Photometric Sunspot Index) was suggested by them. Steinegger et al. (1996) used intensity histograms derived from a photometry of spots, so that not only accurately determined umbra/penumbra areas, but also variations of the spot contrast within the spot enter into the computation of the PSI. Using the empirical coefficients that partly reflect intensity variations among Ca plages by Vršnak et al. (1991), Steinegger et al. (1996) partly improved the PFI function too. However, the $\mu$ dependence (cosine of the heliocentric angle) of spot pixels is lost in this histogram method. The strong variability of the bolometric contrast of sunspots during their disk passage was demonstrated by Chapman et al. (1994). The variability of the three spectral and of the total 
solar irradiance values measured by the VIRGO (Variability of IRradiance and Gravity Oscillations) on board the SOHO was modelled by Fligge et al. (1998). Solanki \& Fligge (2002) analyzed solar magnetograms and could show that over $90 \%$ of the irradiance variations on a solar rotation time scale are due to magnetic features at the solar surface. A review of the short- and long-term variations in the total and spectral irradiances and their relation to the evolution of magnetic fields was published recently by Pap et al. (2002).

In this study, we introduce the starspot modelling approach into solar irradiance studies. The Sun is the only star where surface features can be examined by direct observations. Therefore, unlike the photometric starspot models, which predict the location, size and temperature of pre-assumed spots, our method uses sunspot shapes and positions together with the solar light curves to derive the effective sunspot temperatures. VIRGO on board SOHO supplies the light curves of the Sun in three spectral $(402,500$, and $862 \mathrm{~nm}$ ) and one total (bolometric) irradiance channels since 1996. On the other hand, photometric quality digital images are available from today's PSPT (Precision Solar Photometric Telescope) network and from SOI/MDI (Solar Oscillations Investigation/Michelson Doppler Imager) also on board SOHO (Domingo et al. 1995) to reveal accurate shapes, sizes and locations of sunspots.

When modelling stellar light curves, any brightening effect of faculae is disregarded; only the darkening effect of spots is computed. For the Sun, the effect of faculae is known to be comparable and sometimes even larger than the darkening effect of sunspots (Chapman 1984; Chapman et al. 1982, 1986, 1992; Lawrence 1987; Foukal \& Lean 1988; Steinegger et al. 1996). Faculae have zero or negligible contrast at or near the disk center. Therefore, we have chosen several days around the central meridian passage of a single spot group, NOAA 7981, as a simple case that permits us to compute spot temperatures from VIRGO data. Thus, in this first approach we avoid the complications of faculae. The MDI images of this period are used as templates supplying only the areas and the positions ( $\mu$ values) of spot pixels; the intensity information was only used for discrimination between photospheric and spot pixels - not for the derivation of temperatures.

This study presents a new technique of deriving accurate effective sunspot temperatures from satellite precision photometry of solar irradiance. Unlike previous techniques, it does not require precise contrast measurements of sunspots, which are usually corrupted by an unknown amount of stray light. Moreover, this approach of studying irradiance variations represents a first step towards the disentanglement of spot deficits from facular excess.

\section{Data and analysis procedures}

\subsection{VIRGO data}

For the present analysis we used hourly mean values of the solar spectral irradiance (SSI) measured at 402, 500, and $862 \mathrm{~nm}$ (with a bandwidth of $5 \mathrm{~nm}$ each) and of the total solar irradiance (TSI) as supplied by the VIRGO experiment (TSI data version v3.5). A boxcar average over 23 hourly values was applied to smooth out short-term variations of much less than a day. In this way we obtained a light curve of the Sun for the period from 29 April throughout the end of the year 1996. The procedure applied for an additional detrending of the data was: i) in the given period days of very low solar activity were selected on the conditions that no sunspots were visible, BBSO CaII index was low, MgII core-to-wing ratio was low, and TSI exhibited a relative minimum; ii) in each channel a cubic spline was fit into the 6 points determined in this way; iii) TSI and the three SSI value sets were referred to these spline fits, where the required corrections were usually smaller than $40 \times 10^{-6}$; iv) the spline fits were regarded as the "zero activity lines" and all values were converted into ppm differences with respect to these lines. Figure 1 shows the smoothed hourly means (solar light curves) of the 3 VIRGO spectral channels and the TSI channel for the period 16 July (day 198) to 17 August (day 230) 1996.

\subsection{MDI white-light full disk images}

For this analysis 33 full disk white-light images were retrieved from the MDI database; they cover the period from 26 July to 9 August 1996. An average smoothed center-to-limb variation (CLV) was determined for each image by measuring the mean intensity along concentric rings of constant $\mu$ and fitting a 4th degree polynomial into these means. The fitting procedure was only carried out up to $99.5 \%$ of the solar image radius in order to avoid problems with the steep intensity gradients near the solar limb. Each image was then divided by the smooth CLV which yielded "flat" images in which the quiet Sun photospheric intensity was normalized to unity. From these images the positions of sunspot pixels were determined; all pixels with intensity $\leq 0.92$ were treated as penumbral and umbral areas, and the limit between umbra and penumbra was set to 0.55 of the mean photospheric intensity. Those limits were chosen to get best agreement between umbral and penumbral areas derived from Kanzelhöhe photoheliograms and from the MDI images. They also agree well with those given in the literature on the penumbral intensity range, e.g. Grossmann-Doerth \& Schmidt (1981). It is important to note that nowhere in the following analysis pixel intensities were used directly for the determination of the flux deficit or excess. Figure 2 shows the disk passage of the active region NOAA 7981 for 12 consecutive days, i.e. day 209 to 220 of 1996 while Fig. 3 gives the areas of sunspots and faculae during the same period.

\section{Mean spot temperature}

An algorithm to compute sunspot deficits using digital images of the Sun is presented in Appendix A. The adoption of this algorithm for computing sunspot temperatures using the deficit values (Fig. 1) and sunspot pixel positions (from MDI full disk continuum images) is given in Appendix B (both appendices can be found under http://www.edpsciences.org). The first step is to compute the spot-to-photosphere intensity ratio $(\alpha)$ characterizing the channel of the deficit value used, representing all pixels of a spot group. Note that the 


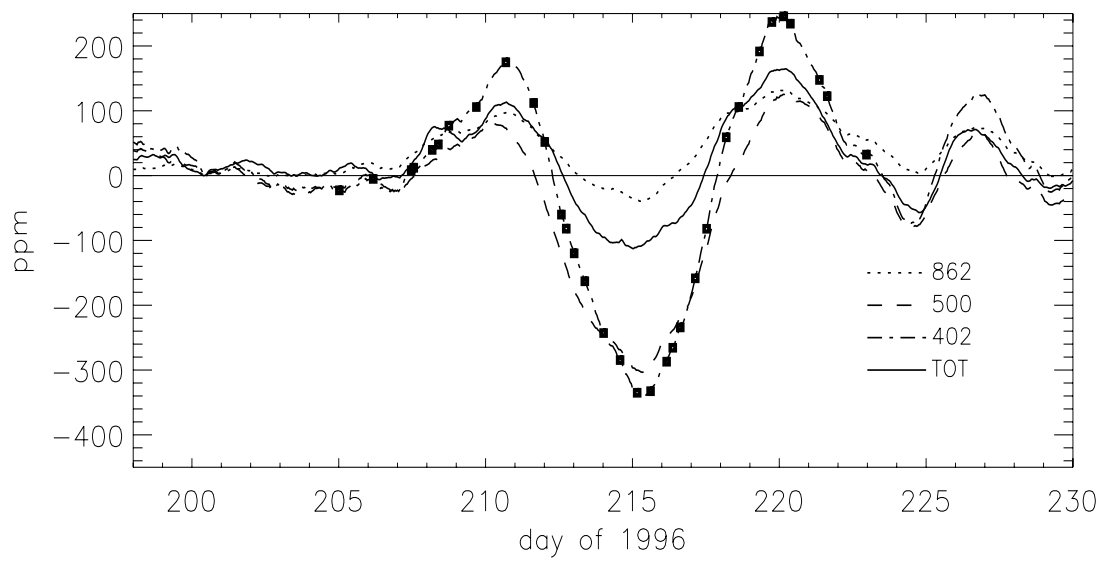

Fig. 1. Smoothed hourly means of the 3 VIRGO spectral irradiances and the total solar irradiance in the period 16 July (day 198 ) to 17 August (day 230) 1996. Filled symbols mark the times of MDI full disk white-light images on the $402 \mathrm{~nm}$ curve.

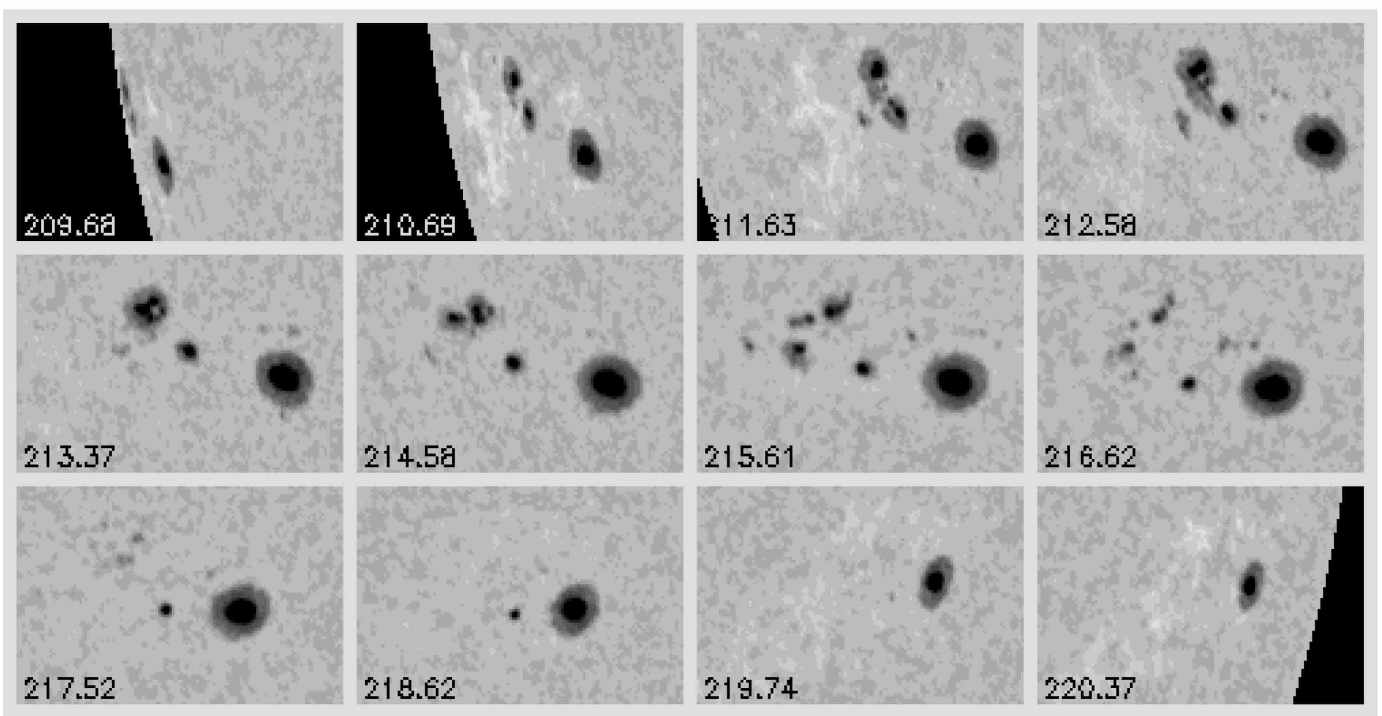

Fig. 2. Disk passage of active region NOAA 7981 from 27 July 1996 (day 209) to 7 August 1996 (day 220); central meridian passage was on 2 August (day 215.6). The times are given as fractions of the day; data are extracted from full disk MDI images.

definition of $\alpha$ used here (i.e. $I_{\mathrm{s}} / I_{\mathrm{p}}$, with $I_{\mathrm{s}}$ being the spot intensity and $I_{\mathrm{p}}$ the photospheric intensity) differs from the definition given in some of the earlier work. Figure 4a displays the computed $\alpha$ values for the four channels of VIRGO during the disk transit of the spot group NOAA 7981. The $\alpha$ values derived directly from MDI images as the mean of intensities $\leq 0.92$ were also plotted for a comparison. The next step is to transform computed $\alpha$ to the temperature differences $\left(T_{\mathrm{p}}-T_{\mathrm{s}}\right)$ between the spot and the local photosphere, which are shown in Fig. 4b.

Eker (1994) assumed $\alpha$ is independent of $\mu$ when modeling stellar light curves. This asumption appears to be confirmed by $\alpha$ of MDI in Fig. 4 a because it stays nearly constant during the disk passage. This, however, does not mean the $T_{\mathrm{p}}-T_{\mathrm{s}}$ must stay constant since the functions for computing $T_{\mathrm{p}}$ and $T_{\mathrm{s}}$ contain the $\mu$ dependent CLV function and their relation to $\alpha$ is not linear. If the spot and the photosphere are viewed at different $\mu$, the different atmospheric heights are observed. With $\mu$ decreasing towards the limb, both $T_{\mathrm{p}}$ and $T_{\mathrm{s}}$ decrease. The solid curve (MDI) in Fig. 4b indicates that the local photospheric temperature decreases more than the mean spot temperature as the spot group comes closer to the limb.

However, the $\alpha$ in the VIRGO channels do not show a tendency of staying constant as the $\alpha$ of MDI. This is because the VIRGO deficit values are affected by the faculae when the active region is sufficiently away from the disk center: the facular emission decreases the deficit. Thus, higher values of $\alpha$ were computed. A few images close to central meridian passage must be free of the effect of faculae according to Fig. $3 \mathrm{~b}$. Therefore, the predicted $\alpha$ and the corresponding $T_{\mathrm{p}}-T_{\mathrm{s}}$ values of those images can be trusted as being free of facular emission.

Zero level problem. According to Fig. $4 \mathrm{~b}$, the $T_{\mathrm{p}}-T_{\mathrm{s}}$ from VIRGO are smaller than those from MDI. Moreover, they spread over a range between the lowest value for the $862 \mathrm{~nm}$ $\left(T_{\mathrm{p}}-T_{\mathrm{s}}=64 \mathrm{~K}\right)$ and the highest value of the $500 \mathrm{~nm}$ channel $\left(T_{\mathrm{p}}-T_{\mathrm{s}}=344 \mathrm{~K}\right)$ on day 215.6. One must expect different values of $T_{\mathrm{p}}-T_{\mathrm{s}}$ at different VIRGO channels since the 

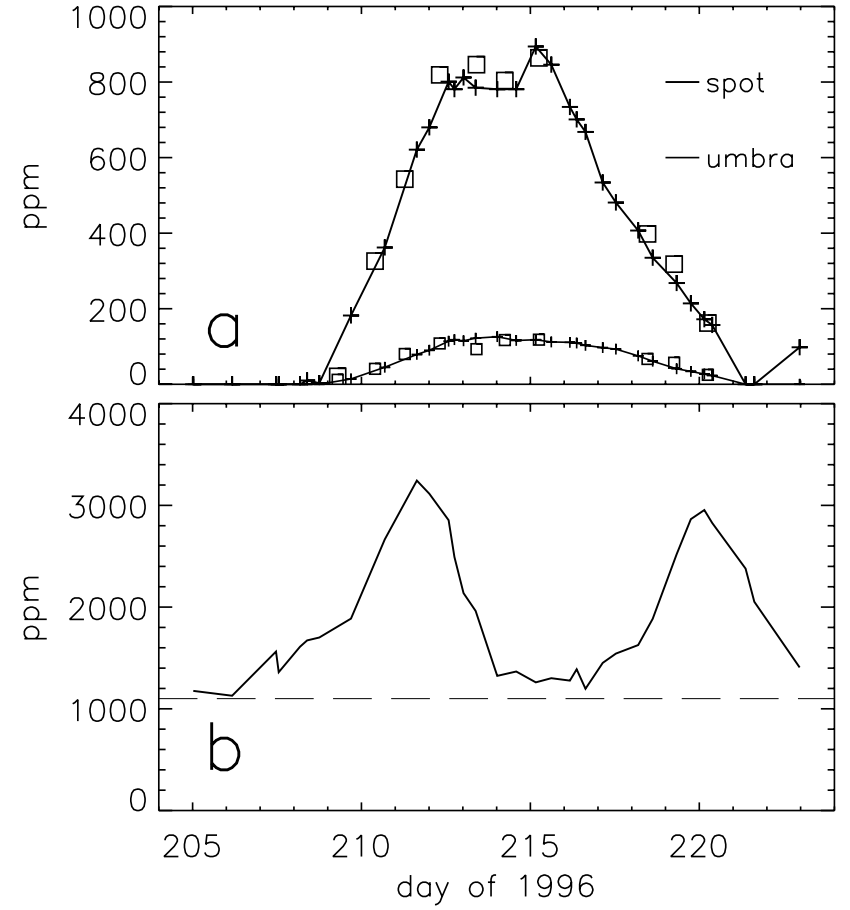

Fig. 3. a) Areas of sunspot group NOAA 7981 (in millionths of the solar disk); + from MDI; $\square$ from Kanzelhöhe photoheliograms; b) facular areas derived from MDI full disk white-light images (in millionths of the solar disk).

brightness temperature at the disk center $\left(T_{0}\right)$ changes because of the wavelength dependent opacity. However, the large scatter found here hints at a problem. The $T_{\mathrm{p}}-T_{\mathrm{s}}$ value at the $862 \mathrm{~nm}$ channel seems to be too low. Too small deficits of this channel in Fig. 1, however, explain the reason. According to Wehrli (1999), the TSI channel of VIRGO is the one producing the most reliable irradiance that the 500 and $402 \mathrm{~nm}$ channels in Fig. $4 \mathrm{~b}$ can be judged as overestimating the $T_{\mathrm{p}}-T_{\mathrm{s}}$ values while the $862 \mathrm{~nm}$ channel underestimates them.

The VIRGO data are normalized with respect to the quiet periods as explained in Sect. 2. Obviously, the zero level obtained from this normalization is not accurate enough to compute the deficit temperatures. We suspect that there still may be a zero level problem inherent in the data. A vertical shift of the data within the uncertainties might produce consistent $T_{\mathrm{p}}-T_{\mathrm{s}}$ values. The amount of the shift at each VIRGO channel is decided after the so called "artificial VIRGO test" described in the following.

Artificial VIRGO test. In the period examined in this study there are 33 MDI images. The beginning and the end of the period is quiet, that is, there are no spots visible on the solar disk. Only 23 images in the middle show the disk passage of the spot group NOAA 7981. These 23 images have been used to produce artificial VIRGO data to conduct a test: what will happen if one under- (or over-)estimates the deficit value due to an error of the zero level?

As the averaged intensities of the spot region (MDI images) stay constant during the disk passage, the values of $\alpha$ for each channel is fixed. The values of $0.85764,0.78693,0.72994$

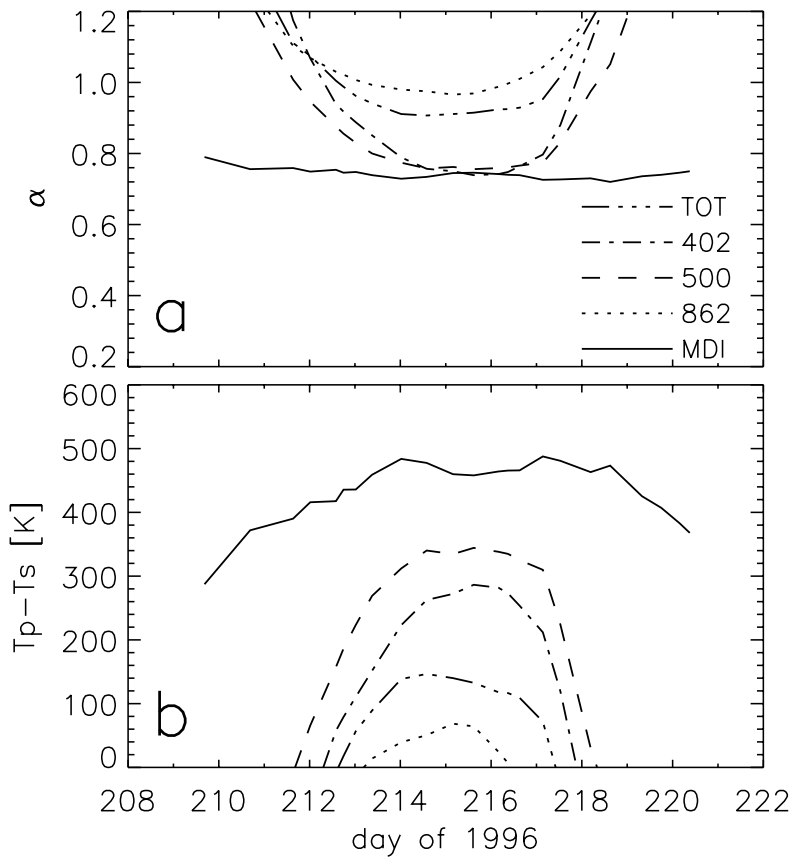

Fig. 4. a) Mean spot-to-photosphere intensity ratios $(\alpha)$ computed from the 402, 500, $862 \mathrm{~nm}$ and TSI channels of VIRGO as well as from the MDI full disk white-light images; b) corresponding temperature differences $\left(T_{\mathrm{p}}-T_{\mathrm{s}}\right.$, where $T_{\mathrm{p}}$ is the local photospheric, $T_{\mathrm{s}}$ is the mean effective spot temperature).

and 0.81732 were computed for the $862,500,402 \mathrm{~nm}$ and the total irradiance, resp., assuming that the spot group is $300 \mathrm{~K}$ cooler than the photosphere at disk center for all channels. The photospheric brightness temperatures of the disk center at the VIRGO channels: 6036, 6178, 5997, and $6100 \mathrm{~K}$ were taken from Landolt-Börnstein (1981). With the above fixed values of $\alpha$, with the CLV coefficients from Neckel \& Labs (1994), and using the $23 \mathrm{MDI}$ images with spots, the light curves of the 4 VIRGO channels (Fig. 5a) have been computed.

Those light curves are used as an artificial data set and are fed to the program which computes $\alpha$ and $T_{\mathrm{p}}-T_{\mathrm{s}}$ in 3 different cases: 1) shifted down to increase the deficits by $100 \mathrm{ppm} ; 2$ ) no shifting; 3) shifted up to decrease the deficits by $100 \mathrm{ppm}$. The results are plotted in Figs. 5b, c. The test shows that overestimating the deficit results in lowering the $\alpha$, thus the spots appear cooler. However, due to foreshortening effects, the same amount of error on a deficit near disk center reflects differently on the computed $\alpha$ than away from disk center. Only without a systematic shift can constant values of $\alpha$ be obtained. In summary, the true zero level of the VIRGO data can be found by shifting the data on vertical axis until the computed values of $\alpha$ become constant, that is parallel to the abscissa. In this way an optimum solution can be found for each channel independently.

The Optimum Solution. If the VIRGO data (Fig. 1) are shifted such that the zero line of each channel touches the top of the left plateau, then the deficits are systematically overestimated by an amount corresponding to the facular contribution at each channel at the time of the hump in the first half of the disk passage. It is known and also indicated by Fig. 1 that the 

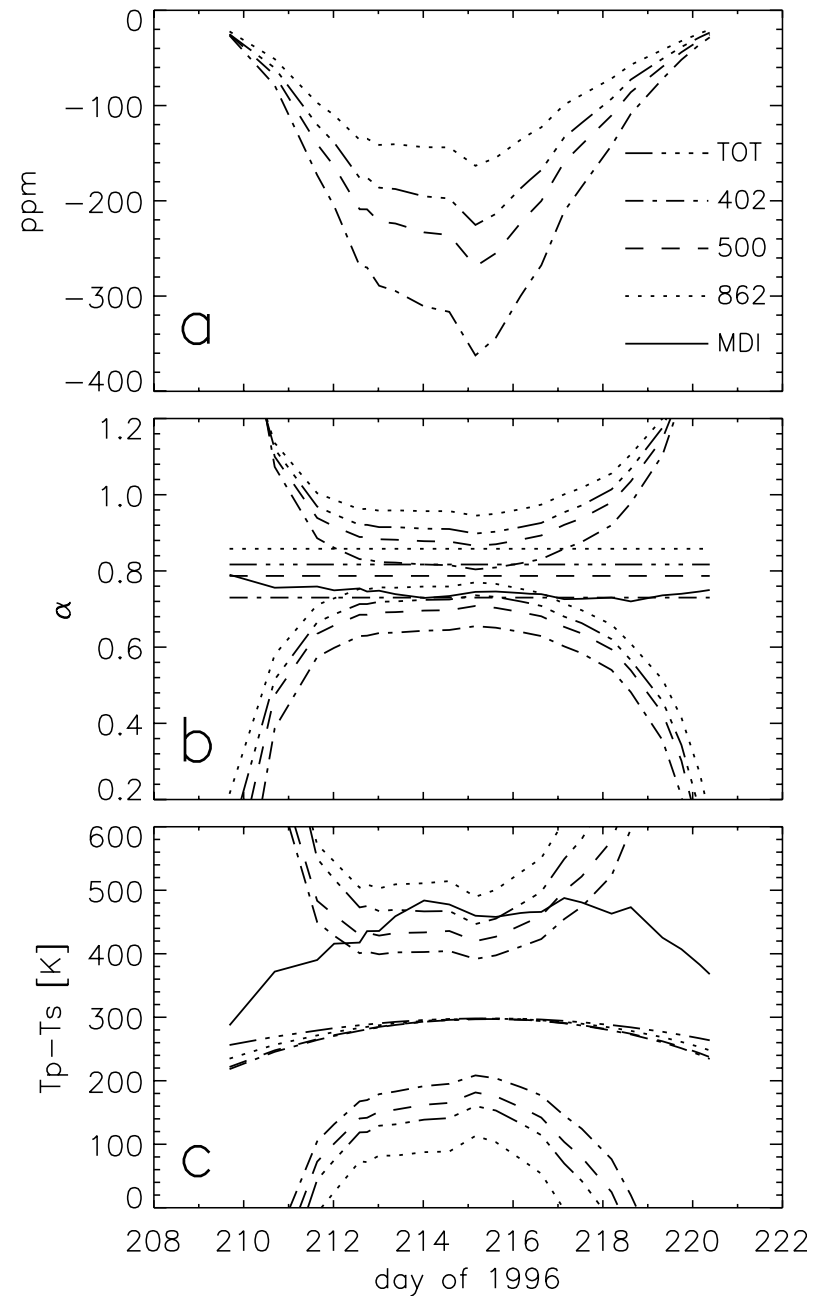

Fig. 5. a) Light curves according to MDI spot areas and locations assuming $300 \mathrm{~K}$ temperature difference between spot and photosphere; b) Mean $\alpha$ recomputed after shifting the zero level of the above light curves by $+100 \mathrm{ppm}$ (set of curves at top), by $0 \mathrm{ppm}$ (center set of curves), and by $-100 \mathrm{ppm}$ (set of curves at bottom); c) Predicted $T_{\mathrm{p}}-T_{\mathrm{s}}$ derived from the curves shown in $\mathbf{b}$ ).

faculae contribute more at shorter wavelengths. Consequently, the channel with the longest wavelength $(862 \mathrm{~nm})$ is expected with the smallest systematic increase, thus, the smallest $T_{\mathrm{p}}-T_{\mathrm{s}}$ (Fig. 6b). Therefore, the maximum of $T_{\mathrm{p}}-T_{\mathrm{s}}$ displayed by the $862 \mathrm{~nm}$ channel, i.e. during the central meridian passage, can be treated as an upper limit to all channels.

The sharp changes of the curvature into the wrong direction are marked by the vertical dashed lines in Fig. 6a. Between these lines, the shapes of the curves are consistent with those in Fig. 5b from the artificial VIRGO test. Outside the period marked by the two dashed lines, the $\alpha$ increases in a way contrary to Fig. 5b. This is due to the facular contribution into deficit values. Because of the systematic shift, the effect of faculae shows up more clearly in Fig. 6a than in Fig. 4a. According to Fig. $6 \mathrm{~b}$ the maximum $T_{\mathrm{p}}-T_{\mathrm{s}}$ in the $862 \mathrm{~nm}$ channel is $252 \mathrm{~K}$ while the maximum in the TSI channel is $328 \mathrm{~K}$. The curvature of the $862 \mathrm{~nm}$ data between the dashed lines, however, is different from that in the other channels as if the $862 \mathrm{~nm}$ deficits are still less than they are supposed to be. Thus, the maximum

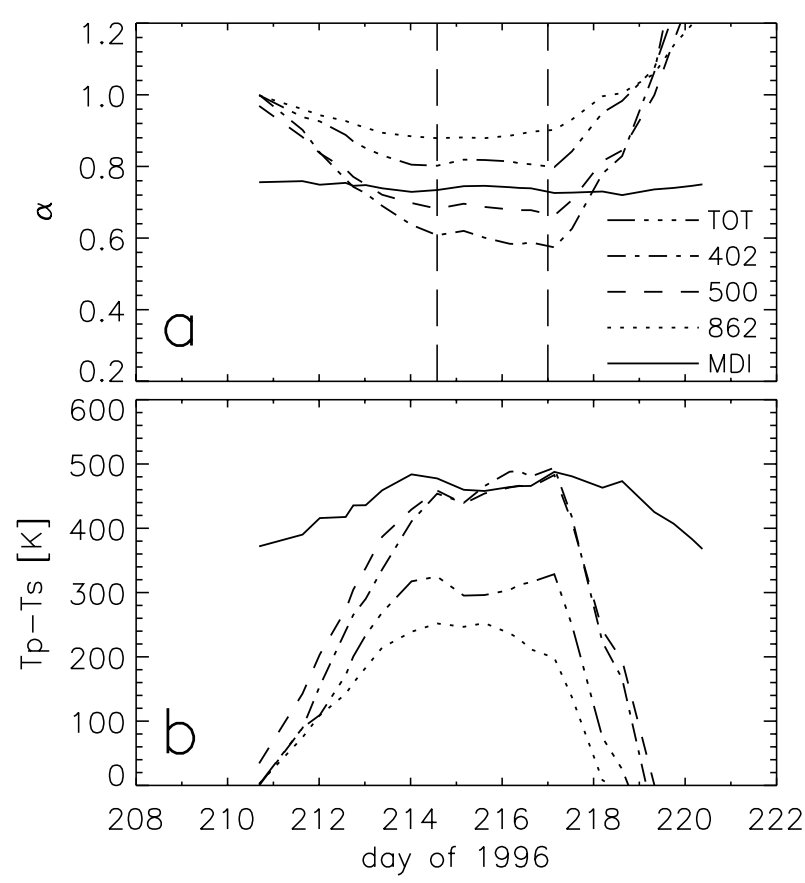

Fig. 6. a) Mean $\alpha$ recomputed after shifts of the zero levels of the VIRGO at each channel separately (see text); b) Predicted $T_{\mathrm{p}}-T_{\mathrm{s}}$ derived from the curves shown in a).

$T_{\mathrm{p}}-T_{\mathrm{s}}$ of the $862 \mathrm{~nm}$ channel might still be less than the true upper limit. Therefore, in the optimum solution, we expect that the $T_{\mathrm{p}}-T_{\mathrm{s}}$ of all channels should not to exceed $300 \mathrm{~K}$.

The optimum solution was searched for in each channel independently as described by the artificial Virgo test. The optimum shifts were found to be: $-97 \mathrm{ppm}$ for the $862 \mathrm{~nm}$, $+30 \mathrm{ppm}$ for the $500 \mathrm{~nm},-10 \mathrm{ppm}$ for the $402 \mathrm{~nm}$, and $-80 \mathrm{ppm}$ for the TSI channel. The optimally shifted data and the results for $\alpha$ and $T_{\mathrm{p}}-T_{\mathrm{s}}$ are displayed in Fig. 7. The values $\alpha=0.879,0.781,0.731$, and 0.847 are predicted from the image on day 215.6 for the $862,500,402 \mathrm{~nm}$ and the TSI channel, respectively. The corresponding $T_{\mathrm{p}}-T_{\mathrm{s}}$ values are $251 \mathrm{~K}$, $306 \mathrm{~K}, 296 \mathrm{~K}$, and $247 \mathrm{~K}$, respectively.

It should be remembered that the results are sensitive to the intensity limit defining the spot pixels on the MDI images. Our limit is $I_{\mathrm{s}} \leq 0.92$ of the local photospheric intensity. Increasing the limit to $I_{\mathrm{s}}<0.95$ increases the number of spot pixels, which in turn, with the same deficit values of VIRGO, it lowers the $T_{\mathrm{p}}-T_{\mathrm{s}}$ values to $213 \mathrm{~K}, 257 \mathrm{~K}, 247 \mathrm{~K}$, and $208 \mathrm{~K}$ respectively.

The spots on the MDI images appear to be much colder than the values derived from the VIRGO data. Fig. 7c implies that the $T_{\mathrm{p}}-T_{\mathrm{s}}$ of MDI images are about $160 \mathrm{~K}$ higher (i.e. the spot cooler) than the $T_{\mathrm{p}}-T_{\mathrm{s}}$ of VIRGO.

\subsection{Umbra and penumbra temperatures}

The mean umbra-to-penumbra contrast ratio, or its reciprocal, is nedded to derive mean umbra and penumbra temperatures (See Appendix B). A digital image may supply it. However, its conversion to the wavelengths of VIRGO channels is essential. 

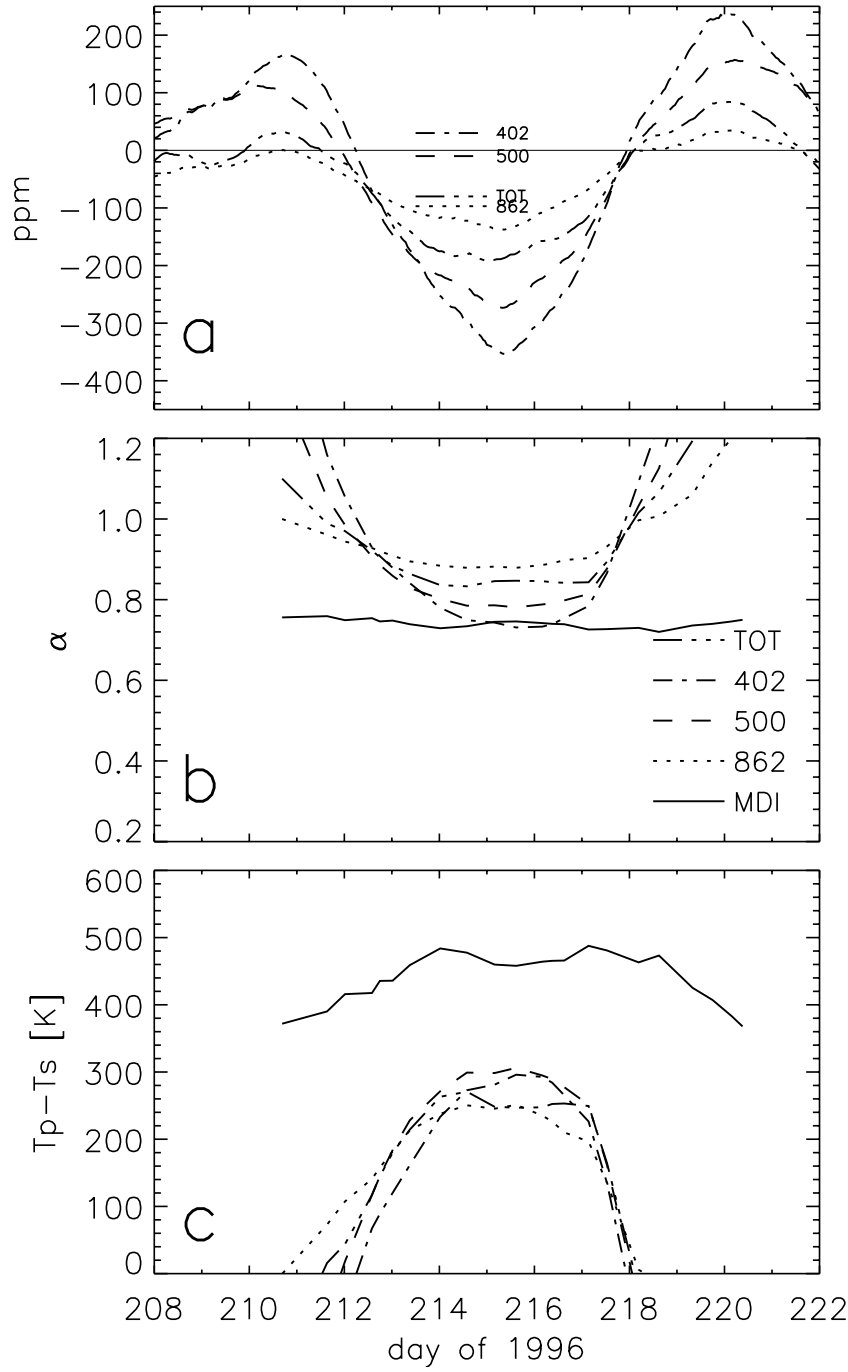

Fig. 7. a) Optimally shifted VIRGO data; Shifts are marked by horizontal lines; b) Mean $\alpha$ after optimum shifts were applied; c) Predicted $T_{\mathrm{p}}-T_{\mathrm{s}}$ derived from the curves shown in $\left.\mathbf{b}\right)$.

Therefore, we have introduced wavelength dependent correction coefficients as

$C_{\lambda}=\frac{\left(\frac{1-\alpha_{\mathrm{u}}}{1-\alpha_{\mathrm{pu}}}\right)_{\lambda}}{\left(\frac{1-\alpha_{\mathrm{u}}}{1-\alpha_{\mathrm{pu}}}\right)_{\mathrm{MDI}}}$

where $\left(\frac{1-\alpha_{\mathrm{u}}}{1-\alpha_{\mathrm{pu}}}\right)_{\lambda}$ and $\left(\frac{1-\alpha_{\mathrm{u}}}{1-\alpha_{\mathrm{pu}}}\right)_{\text {MDI }}$ are the umbra-to-penumbra contrast ratios at a VIRGO channel and at the MDI wavelength. Using the definition of $\alpha$ in the Appendix B (B.9), it is possible to compute the contrast ratios and then $C_{\lambda}$ by a Planck function and the mean umbra/penumbra temperatures. Nevertheless, we preferred not to use the MDI images to derive the umbra and penumbra temperatures because the previous whole spot solution already indicated that the MDI spot intensities are too low which results in too low spot temperatures. Instead, we preferred to go through an iteration procedure to obtain $C_{\lambda}$ together with the local umbral and penumbral temperatures. The iteration is done only once using the image of day 215.6 which we call the calibration image. It is one of the images between
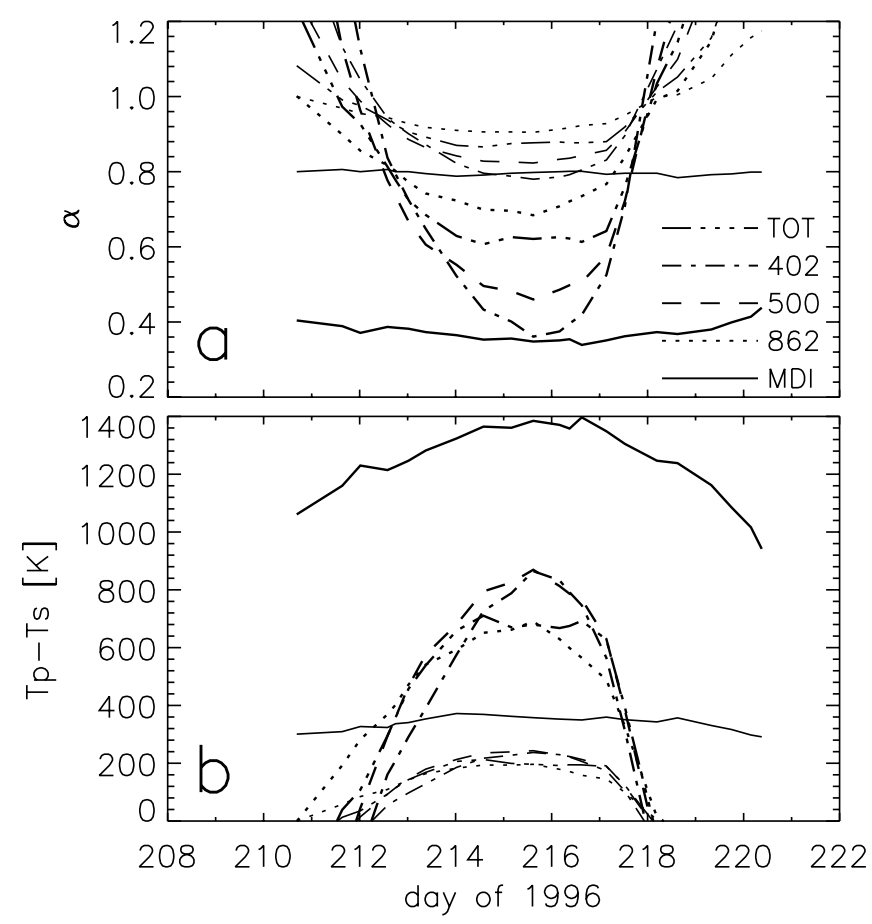

Fig. 8. a) Mean $\alpha$ for the umbra (thick) and penumbra (thin); b) Mean umbra-to-photosphere (thick) and penumbra-to-photosphere (thin) temperature differences.

the vertical dotted lines in Fig. 6a which provides the smallest $\alpha$ values.

The procedure is described as follows assume $C_{\lambda}=1$ for all channels and use the umbra-penumbra contrast ratio of the calibration image in computing $T_{\mathrm{u}}, T_{\mathrm{pu}}$ and $T_{\mathrm{p}}$ for all channels. There are four channels, thus there will be only four values of each temperature which are to be averaged to obtain mean values of $T_{\mathrm{u}}, T_{\mathrm{pu}}$ and $T_{\mathrm{p}}$. Then, the new cotrast ratios are calculated at the wavelengths of all channels including the wavelength of the MDI images. Finally, from Eq. (1) proper values of $C_{\lambda}$ are computed. This is the end of the first iteration. Newly computed values of $C_{\lambda}$ are used together with the original contrast ratio from the calibration image to obtain new $T_{\mathrm{u}}$, $T_{\mathrm{pu}}$ and $T_{\mathrm{p}}$ which refreshes the value of $C_{\lambda}$. After a few steps the iteration settles to unchanging values and is stopped. The obtained $C_{\lambda}$ values then are used for all other images because $\alpha$ is independent of $\mu$.

The solutions for the umbra and the penumbra are displayed in Fig. 8. If the wavelength-dependent correction coefficients $\left(C_{\lambda}\right)$ are not introduced, the derived temperatures become widely scattered. For example, for the $402 \mathrm{~nm}$ channel $T_{\mathrm{p}}-T_{\mathrm{u}}$ becomes as high as $1700 \mathrm{~K}$.

According to the solutions for the day of the calibration image (day 215.6), the temperature of the umbra is $690 \mathrm{~K}, 869 \mathrm{~K}$, $865 \mathrm{~K}$, and $680 \mathrm{~K}$ at the $862,500,402$, and the total channel, respectively. On the other hand, the penumbra solutions yield: $196 \mathrm{~K}, 243 \mathrm{~K}, 237 \mathrm{~K}$, and $196 \mathrm{~K}$ for these four channels. Similar to the whole spot solution, the average intensities from the MDI images are marked in Fig. 8 for comparison. The MDI images again imply a cooler umbra $\left(T_{\mathrm{p}}-T_{\mathrm{u}}=1385 \mathrm{~K}\right)$ and cooler penumbra $\left(T_{\mathrm{p}}-T_{\mathrm{pu}}=358 \mathrm{~K}\right)$. 


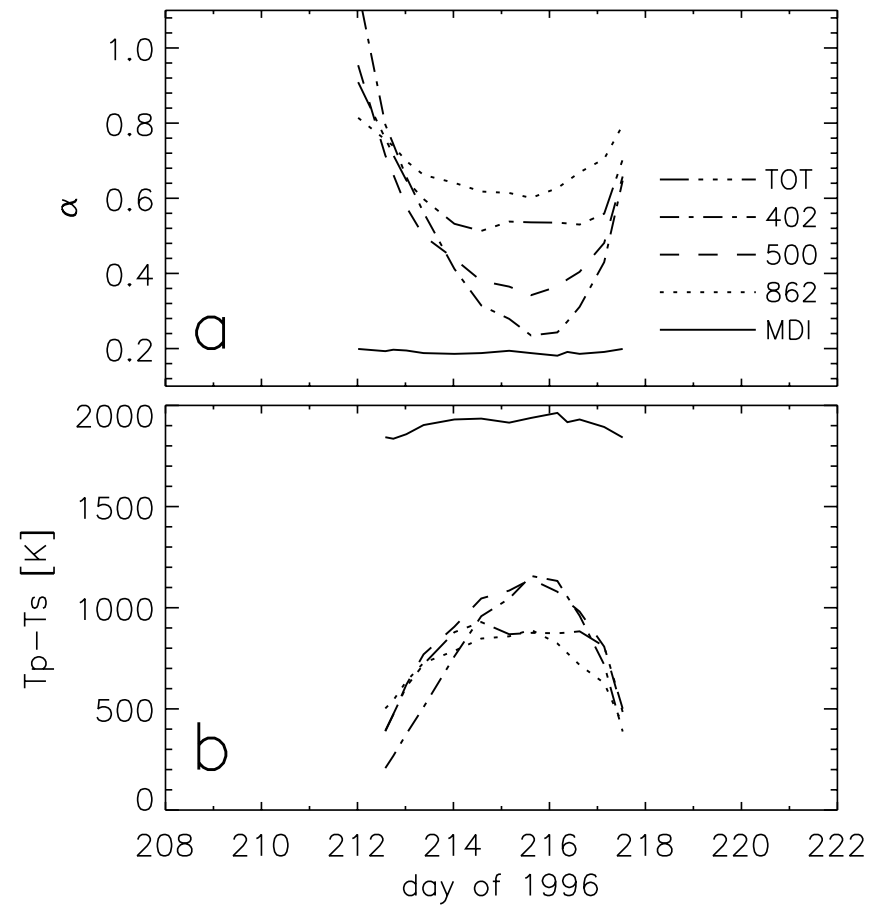

Fig. 9. a) Mean $\alpha$ for the lowest intensity bin (0.15 to 0.20 of $\left.I_{\mathrm{ph}}\right)$ of the spot; b) Corresponding $T_{\mathrm{p}}-T_{\mathrm{s}}$ values.

\subsection{Multilevel solution}

In order to achieve detailed presentation of the spot temperatures, solutions for multiples of 5\% levels of the local photospheric intensity were investigated. In the calibration image (at day 215.6), where the darkest umbral pixels exhibited 0.17 of the photospheric intensity, 16 sublevels with the following limits were chosen: 0.15 to $0.20,0.20$ to $0.25, \ldots, 0.85$ to 0.90 , 0.90 to 0.92 . Note that those intensity limits are used only to discriminate between the levels like in the umbra/penumbra case before. Only the solutions for the coolest level ( $\alpha$ and $T_{\mathrm{p}}-T_{\mathrm{s}}$ ) are shown in Figs. 9a,b. The darkest pixels are $891 \mathrm{~K}$, $1141 \mathrm{~K}, 1157 \mathrm{~K}$, and $876 \mathrm{~K}$ cooler than the local photosphere at the wavelengths $862,500,402 \mathrm{~nm}$ and in the TSI channel of VIRGO. The MDI image, however, appears too cold with 1938 K difference. Solutions of the darkest level do not exist for several images at the beginning and at the end of the period. One possibility is that, due to the Wilson effect, the coolest region of the spot group was hidden.

Replacing the computed $\alpha$ with the intensity of the spot pixels on the MDI images, artificial images can be obtained. Intensity profiles from the row of pixels passing through the spot's minimum pixel of the calibration image (day 215.6) are plotted in Fig. 10 for the two-level and multilevel artificial images. The profiles of the original image are also plotted for a comparison.

In principle, the number of intensity levels may be increased up to the total number of spot pixels if the computing capacity permits. Then, not only level-to-level, but also pixel-to-pixel transformation of the VIRGO data into an artificial digital image becomes possible. Moreover, multiple

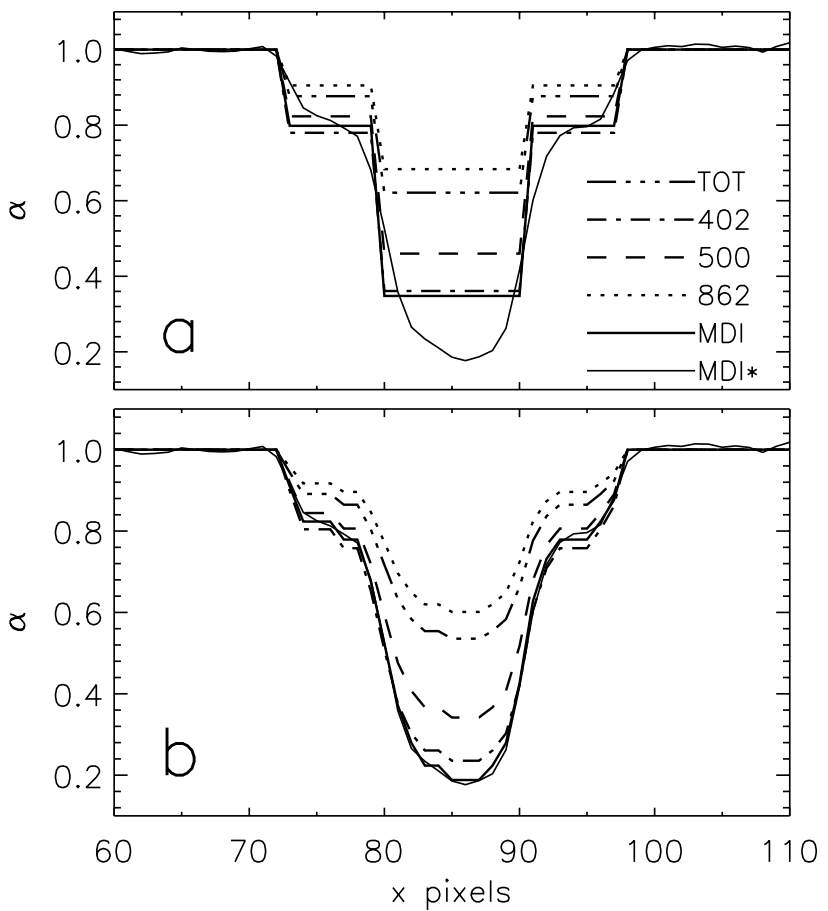

Fig. 10. a) Predicted intensity profiles of the row of pixels through the minimum intensity of the spot on day 215.6; solution for the two-level spot; b) Same for the multilevel spot.

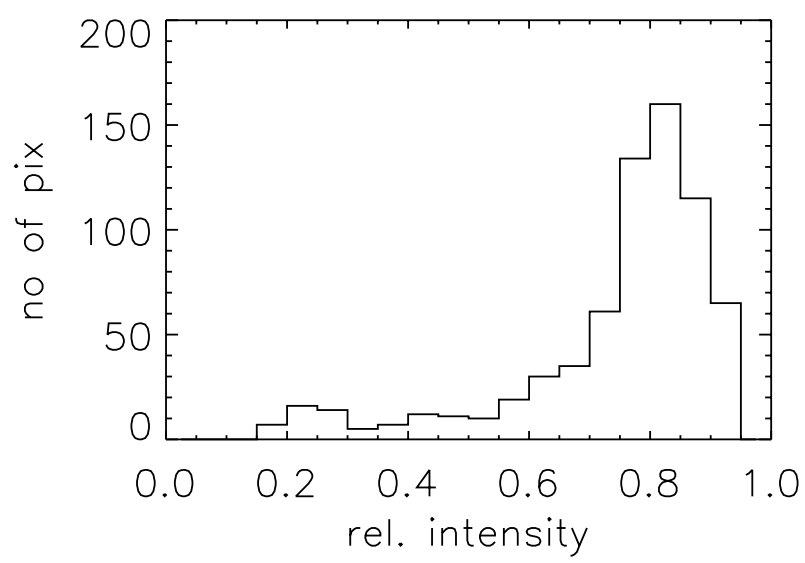

Fig. 11. Histogram of spot pixels for MDI image of day 215.6. Note that the pixel number in the bin $0.90-0.95$ refers to the intensity range $0.90-0.92$.

level solutions may be reduced to give whole spot or umbra/penumbra solutions. (See Appendix B)

Combining the first 8 levels of the multilevel solution on the day of the calibration image, $T_{\mathrm{p}}-T_{\mathrm{s}}=196 \mathrm{~K}, 243 \mathrm{~K}$, $237 \mathrm{~K}$, and $196 \mathrm{~K}$ were obtained for the penumbral pixels from the $862,500,402 \mathrm{~nm}$ and the bolometric channel of VIRGO. Similarly, $T_{\mathrm{p}}-T_{\mathrm{s}}=690 \mathrm{~K}, 870 \mathrm{~K}, 867 \mathrm{~K}$, and $681 \mathrm{~K}$ for the umbral pixels. The difference from the direct solution of the umbra/penumbra case is $<1 \mathrm{~K}$. if the whole spot solutions are compared, both solutions are in agreement to better than $0.1 \mathrm{~K}$, with values $T_{\mathrm{p}}-T_{\mathrm{s}}=251.2 \mathrm{~K}, 306.1 \mathrm{~K}, 296 \mathrm{~K}$, and $246.8 \mathrm{~K}$ at the four channels of VIRGO.

In order to examine the ratio of the umbral area to penumbral area, we have computed the histogram of the spot pixels for 
NOAA 7981 for day 215.6 (cf. Fig. 2). It is shown in Fig. 11 and makes it clear that the penumbral area dominates by a large factor over the umbral area, which explains why the mean effective spot temperatures are $\simeq 300 \mathrm{~K}$.

\section{Discussion and conclusion}

\subsection{VIRGO channels are independent}

It has been shown that a mean effective spot temperature can be computed independently for each of the four channels of VIRGO. Only three pieces of information are sufficient to calculate the mean effective spot temperature:

- 1) a digital image allowing $\mu$ of the spot pixels,

- 2) the CLV of the photosphere,

- 3) the irradiance deficit value.

Unfortunately, two or more irradiance deficit values at different wavelengths do not help to gain extra information, i.e. mean umbra and penumbra temperatures without the external information on the umbra/penumbra contrast ratio. Even if the um$\mathrm{bra} /$ penumbra area ratio from the digital images are available, it does not help to eliminate the necessity of this external information. This is not the weakness of our method. This is the nature of the problem. See the proof in Appendix B.

\subsection{Excess radiation in the red (862 $\mathrm{nm})$ and bolometric VIRGO channels}

For the period considered in this study, we were unable to compute consistent temperature deficits $\left(T_{\mathrm{p}}-T_{\mathrm{s}}\right)$ from the normalized and de-trended VIRGO data. Consistent $\left(T_{\mathrm{p}}-T_{\mathrm{s}}\right)$ values were only achieved by shifting the zero levels of the VIRGO. The shifts $(-97 \mathrm{ppm}$ for the red $(862 \mathrm{~nm}),+30 \mathrm{ppm}$ for the green $(500 \mathrm{~nm}),-10 \mathrm{ppm}$ for the blue $(402 \mathrm{~nm})$, and $-80 \mathrm{ppm}$ for the bolometric channel) were obtained manually by trial and error - but according to the principle that the predicted alphas must be constant during the period of the spot group's central meridian passage, when the facular contribution is negligible.

The VIRGO values during the quiet period before the active region NOAA 7981 appeared on the solar disk suggest that the shifts applied to the 500 and to the $402 \mathrm{~nm}$ data set are acceptable within the uncertainty of the data according to a visual inspection of Figs. 1 and 7a. Our error analysis and the artificial VIRGO test indicate that about $40 \mathrm{ppm}$ uncertainty of the data propagates into about $60 \mathrm{~K}$ uncertainty in the $T_{\mathrm{p}}-T_{\mathrm{s}}$ values. Therefore, the predicted values of the whole spot solution are expected to be consistent within $\pm 60 \mathrm{~K}$ uncertainty.

The red and the bolometric channels of VIRGO appear to be polluted by an excess emission even during the central meridian passage of the spot group. Indeed, the unshifted red data on Fig. 1 exhibits abnormally weak deficits. Thus, an abnormally large excess emission (above the zero level line) as compared to the deficit values in the middle of the period is obvious. We cannot rule out the possibility that the strong $\mathrm{Ca}^{++}$ line at $866.217 \mathrm{~nm}$, i.e. less than $5 \mathrm{~nm}(1 F W H M$ of the filter passband) to the red of the center wavelength of the $862 \mathrm{~nm}$ filter, contributes to this excess radiation. The excess emission in the red at the middle of the period could also be explained if faculae contribute at the center of the disk. Thus the red curve of VIRGO is distorted.

The total irradiance behaves similarly to the red channel. Since this channel integrates over all wavelengths, it is absurd to think of a leakage as in the red channel. It seems that whatever affects the red channel similarly affects the total channel. If the excess emission at the red filter is explained by a leakage of the red filter, then how could the excess emission in the total channel be explained? The idea of optically thick faculae at longer wavelengths fits better into the picture to explain the excess radiation in both the red and the total channels - unless the $402 \mathrm{~nm}$ and the $500 \mathrm{~nm}$ channels are proven to overestimate the deficit values. Due to the curvature of the red data in Fig. 6a, the red data deficits seem to be underestimated even if the zero level line is moved on the left shoulder. This, however, implies that even if the excess emission in the total channel is explained by optically thicker faculae at longer wavelengths, the possibility of a leakage in the red channel cannot be ruled out.

\subsection{Sunspots and starspots}

Long before the solar variability was discovered, sunspots inspired stellar astronomers to explain brightness variations of some stars. In fact, the starspot hypothesis was the first idea to explain brightness variations of the first variable stars discovered (cf. e.g. the reviews by Kopal 1982, Hall 1994). But, because of the uniqueness problem attributed to spot solutions, and because other types of variabilities, such as eclipses, pulsations etc., were discovered, the starspot hypothesis was discarded during the first half of the 20th century. However, in the second half, especially after the seventies, quantitative starspot models started to thrive because other physical effects (eclipses, pulsations, etc.) turned out to be inadequate to explain light variations of some stars which nowadays are believed to have spots.

According to Eker (1996, 1999a,b), the starspot hypothesis is consistent and physically meaningful because it is possible to produce synthetic light curves from the physical parameters of spots and it is also possible to recover those parameters from the observed light curves alone. There is no uniqueness problem special to the starspot hypothesis. The problem was that the observed light curves were never sufficiently accurate to allow a successful modelling to resolve more than two, rarely three, spots. Eker (1999a) predicts that about \pm 0.0001 magnitude or better accuracy is needed in photometric observations for a succesfull modelling.

The TSI channel of VIRGO supplies the most accurate intensity measurements of the SUN as a star. According to Wehrli (1999), the accuracy of the TSI channel is about $40 \mathrm{ppm}$ or better. The other channels $(402,500$ and $862 \mathrm{~nm})$ are less accurate. The $40 \mathrm{ppm}$ translates to 0.04 millimagnitude on the magnitude scale. Thus, the present solar light curves meet the requirements of a successful modelling. But still, the classical photometric starspot models are not applicable to the solar light curves for two reasons: 1) The brightening effect of faculae has not yet been included in the starspot models. However, the 
effect of faculae is known to be comparable and sometimes even larger than the darkening effect of sunspots (Chapman 1984; Chapman et al. 1982, 1986, 1992; Lawrence 1987; Foukal \& Lean 1988; Steinegger et al. 1996). 2) There is morphological evolution of sunspots. It is known that some sunspots form and disappear in a single rotation period. Without a priori information how the shape and intensity distribution within a spot evolve, the prediction of spots from the light curves becomes impossible.

Only with a priori assumptions, e.g. a fixed number (1 or 2, rarely 3 ) of spots of unchanging shape (e.g. circular) with uniform temperature, if phase coverage and accuracy is sufficient, the observed light curves of spotted stars allow us to determine size, location and temperature of the presumed spots. Since the spot parameters (size, location, shape, temperature) are mutually dependent, the values predicted at the end are highly model-dependent (Eker 1999b). Vogt (1981) claimed an unambiguous determination of spot temperatures regardless of the shapes and the distribution of spots. According to Vogt, a single circular spot may represent all possibilities. However, the strong model dependence of spot solutions falsifies this claim. The truth is: it is not possible to obtain a spot temperature independent from the other parameter values. In other words, unless accurate geometrical parameters of spots are given or assumed, the light curves cannot produce reliable spot temperatures.

The amplitude of the spotted star light curves is of the order of one tenth of a magnitude. There could be unusual stars like HD12545 which were known to show as much as $0.6 \mathrm{mag}$ (Nolthenius 1991). Eker (1995) found the spots to be $1280 \mathrm{~K}$ cooler than $T_{\text {eff }}=4820 \mathrm{~K}$ of this cool giant covering $27 \%$ of the total surface from the light curves of $\Delta V=0.5 \mathrm{mag}$ amplitude.

Due to large amplitude of the light curves, the starspots were found to be much bigger ( $\approx 10$ times) than sunspots. On the other hand, the temperatures of starspots were predicted to be like those of sunspot umbrae. The effective temperature of the spot group NOAA 7981 was found in this study to be $\approx 300 \mathrm{~K}$ lower than the solar photosphere. This result tells us that the starspot models are not only predicting starspots much larger than sunspots, but also much cooler.

The darkening effect of sunspots can be modeled accurately. The accuracy depends on the solar light curves and the digital image to identify spot pixels. However, the brightening effect of solar faculae is not easy to include due to the lack of a reliable facular model. "Although a considerable amount of information exists about the total and the spectral irradiance variations of the Sun, the underlying physical mechanisms are not well understood as yet. Due to the lack of adequate quantitative physical models one has to rely on empirical models based on proxy indicators of solar activity" (Pap et al. 1994). Therefore, we have preferred to avoid the facular effect in this study. In a forthcoming study the effect of faculae will be analyzed in connection with an investigation of facular models suggested by Eker (2003).

\section{References}

Brandt, P. N., Schmidt, W., \& Steinegger, M. 1990, Sol. Phys., 129, 191

Brandt, P. N., Schmidt, W., \& Steinegger, M. 1992, in Proc. Workshop on the Solar Electromagnetic Radiation Study for Solar Cycle 22, ed. R. F. Donnelly (Boulder, CO, Space Environmental Lab., NOAA ERL), 130

Chapman, G. A. 1984, Nature, 308, 252

Chapman, G. A. 1987, ARA\&A, 25, 633

Chapman, G. A., Cookson, A. M., \& Dobias, J. J. 1994, ApJ, 432, 403

Chapman, G. A., Herzog, A. D., \& Lawrence, J. K. 1986, Nature, 319, 654

Chapman, G. A., \& Meyer, A. D. 1986, Sol. Phys., 103, 21

Chapman, G. A., Herzog, A. D., Lawrence, J. K., \& Shelton, J. C. 1982, ApJ, 282, L99

Chapman, G. A., Herzog, A. D., Lawrence, J. K., \& Walton, S. R. 1992, J. Geophys. Res., 97, 8211

Domingo, V., Fleck, B., \& Poland, A. I. 1995, Sol. Phys., 162, 1

Eker, Z. 1994, ApJ, 420, 373

Eker, Z. 1995, ApJ, 445, 526

Eker, Z. 1996, ApJ, 473, 388

Eker, Z. 1999a, ApJ, 512, 386

Eker, Z. 1999b, New Astron., 4, 365

Eker, Z. 2003, Sol. Phys., 212, 277

Eker, Z., \& Al-Malki, M. 1999, Ap\&SS, 262, 411

Fligge, M., Solanki, S. K., Unruh, Y. C., Fröhlich, C., \& Wehrli, Ch. 1998, A\&A, 335, 709

Foukal, P. V. 1981, in Physics of Sunspots, ed. L. Cram, \& J. H. Thomas (New Mexico: Sacramento Peak Observatory, Sunspot), 391

Foukal, P. V., \& Lean, J. L. 1988, ApJ, 328, 347

Fröhlich, C. 1977, in The Solar Output and Its Variations, ed. O. R. White (Boulder: Colorado Assoc. Univ. Press), 93

Fröhlich, C. 1994, in The Sun as a Variable Star, ed. J. M. Pap, C. Fröhlich, H. S. Hudson, \& S. K. Solanki (Cambridge Univ. Press), 28

Fröhlich, C., Foukal, P. V., Hickey, J. R., Hudson, H. S., \& Willson, R. C. 1991, in The Sun in Time, ed. C. Sonnet, M. S. Giampapa, $\&$ M. S. Matthews (Tucson: Univ. Arizona Press), 11

Grossmann-Doerth, U., \& Schmidt, W. 1981, A\&A, 95, 366

Hall, D. S. 1994, Int. Amat. Prof. Photoel. Photom. Commun. No: 54,1

Hudson, H. S. 1988, ARA\&A, 26, 473

Hudson, H. S., Silva, S., Woodard, M., \& Willson, R. C. 1982, Sol. Phys., 76, 211

Kopal, Z. 1982, Ap\&SS, 87, 149

Kyle, H. L., Hoyt, D. V., \& Hickey, J. R. 1994, Sol. Phys., 152, 9

Landolt-Börnstein 1981, in Astronomy and Astrophysics, Vol. 2a, ed. K. Schaifers, \& H. H. Voigt (Berlin: Springer-Verlag), 90

Lawrence, J. K. 1987, Sol. Phys., 110, 73

Neckel, H., \& Labs, D. 1994, Sol. Phys., 153, 91

Newkirk, G. 1983, ARA\&A, 21, 429

Nolthenius, K. 1991, Inf. Bull. Var. Stars, No. 3589

Pap, J. M., Turmon, M., Floyd, L., Fröhlich, C., \& Wehrli, Ch. 2002, Adv. Space Res., 29, 1923

Pap, J. M., Willson, R. C., Fröhlich, C., Donnelly, R. F., \& Puga, L. 1994, Sol. Phys., 152, 13

Sofia, S., Oster, L., \& Schatten, K. 1982, Sol. Phys., 80, 87

Steinegger, M., Vazquez, M., Bonet, J. A., \& Brandt, P. N. 1996, ApJ, 461,478

Solanki, S. K., \& Fligge, M. 2002, Adv. Space Res., 29, 1933

Vogt, S. S. 1981, ApJ, 250, 327

Vršnak, B., Plačko, D., \& Ruždjak, V. 1991, Sol. Phys., 162, 101

Wehrli, C. 1999, private communication

Willson, R. C., \& Hudson, H. S. 1991, Nature, 351, 42 